\title{
Analysis and Numerical Approximation of an Electro-elastic Frictional Contact Problem
}

\author{
El. Essoufi, El. Benkhira and R. Fakhar * \\ University Hassan I, FSTS, Department of Mathematics and Informatics, \\ B.P. 577, 26000 Settat, Morocco
}

\begin{abstract}
We consider the problem of frictional contact between an piezoelectric body and a conductive foundation. The electro-elastic constitutive law is assumed to be nonlinear and the contact is modelled with the Signorini condition, nonlocal Coulomb friction law and a regularized electrical conductivity condition. The existence of a unique weak solution of the model is established. The finite elements approximation for the problem is presented, and error estimates on the solutions are derived.
\end{abstract}

Key words: piezoelectric, Coulomb's law, Signorini condition, fixed point process, finite element approximation, error estimates

AMS subject classification: 35J85, 47J20, 49J40, 74F15, 74G30, 74M10, 74M15, 74S05

\section{Strong and weak formulation of the mathematical model}

In this section, we state the model of equilibrium process of the elastic piezoelectric body in frictional contact with a conductive deformable foundation and give his weak formulation. We consider an elastic piezoelectric body which initially occupies open bounded subset $\Omega \subset \mathbb{R}^{d}, d=2,3$ with a sufficiently regular boundary $\partial \Omega=\Gamma$. We decompose $\Gamma$ into three open disjoint parts $\Gamma_{1}$, $\Gamma_{2}$ and $\Gamma_{3}$, on the one hand, and a partition of $\Gamma_{1} \cup \Gamma_{2}$ into two open parts $\Gamma_{a}$ and $\Gamma_{b}$, on the other hand, such that meas $\Gamma_{1}>0$ and meas $\Gamma_{a}>0$. The summation convention over repeated indices is used, all indices take values in $\{1, \ldots, d\}$. Everywhere below we use $\mathbb{S}^{d}$ to denote the space of second order symmetric tensors on $\mathbb{R}^{d}$ while " . "and $\|\cdot\|$ will represent the inner product and the Euclidean norm on $\mathbb{R}^{d}$ and $\mathbb{S}^{d}$, that is $\forall u, v \in \mathbb{R}^{d}, \forall \sigma, \tau \in \mathbb{S}^{d} u \cdot v=u_{i} \cdot v_{i}, \quad\|v\|=$ $(v \cdot v)^{1 / 2}$ and $\sigma \cdot \tau=\sigma_{i j} \cdot \tau_{i j}, \quad\|\tau\|=(\tau \cdot \tau)^{1 / 2}$. Moreover, we denote by $u: \Omega \rightarrow \mathbb{R}^{d}$ the

\footnotetext{
${ }^{*}$ Corresponding author. E-mail: rachidfakhar@yahoo.fr
} 
displacement field, $\varepsilon(u)=\left(\frac{1}{2}\left(u_{i, j}+u_{j, i}\right)\right)$ the small strain tensor, $\sigma: \Omega \rightarrow \mathbb{S}^{d}$ the stress tensor, $D: \Omega \rightarrow \mathbb{R}^{d}$ the electric displacement field, $E(\varphi)=-\nabla \varphi$ the electric vector field, where $\varphi: \Omega \rightarrow \mathbb{R}$ is an electric potential. We also use the notations for normal and tangential components of displacement vector and stress: $v_{\nu}=v \cdot \nu, \quad v_{\tau}=v-v_{\nu} \nu, \quad \sigma_{\nu}=(\sigma \nu) \cdot \nu, \quad \sigma_{\tau}=\sigma \nu-\sigma_{\nu} \nu$, where $\nu$ denote the outward normal vector on $\Gamma$. The static equilibrium of the elastic piezoelectric body is described by the following strong equations

$$
\operatorname{Div} \sigma+f_{0}=0, \quad \operatorname{div} D=q_{0}, \quad \text { in } \Omega,
$$

where $\operatorname{Div} \sigma=\left(\sigma_{i j, j}\right)$, and $\operatorname{div} D=\left(D_{j, j}\right), f_{0}$ and $q_{0}$ the density of volume forces and volume electric charges, respectively. The constitutive laws of material is assumed of the form (see [3])

$$
\sigma=\mathcal{F} \varepsilon(u)-\mathcal{E}^{*} E(\varphi), \quad D=\mathcal{E} \varepsilon(u)+\beta E(\varphi) \text { in } \Omega,
$$

in which $\mathcal{F}: \Omega \times \mathbb{S}^{d} \rightarrow \mathbb{S}^{d}$ is a nonlinear elasticity operator, $\mathcal{E}: \Omega \times \mathbb{S}^{d} \rightarrow \mathbb{R}^{d}$ is a linear piezoelectric operator, $\mathcal{E}^{*}: \Omega \times \mathbb{R}^{d} \rightarrow \mathbb{S}^{d}$ is its transpose given by $\mathcal{E} \sigma v=\sigma \mathcal{E}^{*} v, \forall \sigma \in \mathbb{S}^{d}, v \in \mathbb{R}^{d}$ and $\beta: \Omega \times \mathbb{R}^{d} \rightarrow \mathbb{R}^{d}$ is a linear electric permittivity operator.

To complete the model, we have to prescribe the mechanic and electric boundary conditions. According to the physical setting, we use

$$
u=0 \quad \text { on } \Gamma_{1}, \quad \sigma \nu=f_{2} \quad \text { on } \Gamma_{2}, \quad \varphi=0 \quad \text { on } \Gamma_{a}, \quad D \cdot \nu=q_{2} \quad \text { on } \Gamma_{b},
$$

where $f_{2}$ and $q_{2}$ the density of tractions and surface electric charges, respectively. On the contact surface $\Gamma_{3}$, we model the contact with the Signorini condition, the regularized Coulomb law and the regularized electrical conductivity condition, that is

$$
\left.\begin{array}{l}
\sigma_{\nu}(u) \leq 0, u_{\nu} \leq 0, \sigma_{\nu}(u) u_{\nu}=0, \\
\left|\sigma_{\tau}\right| \leq \mu\left(\left\|u_{\tau}\right\|\right)\left|R \sigma_{\nu}(u)\right|, \quad\left\{\begin{array}{l}
\left|\sigma_{\tau}\right|<\mu\left(\left\|u_{\tau}\right\|\right)\left|R \sigma_{\nu}(u)\right| \Rightarrow u_{\tau}=0 \\
\left|\sigma_{\tau}\right|=-\mu\left(\left\|u_{\tau}\right\|\right)\left|R \sigma_{\nu}(u)\right| \frac{u_{\tau}}{\left\|u_{\tau}\right\|} \Rightarrow u_{\tau} \neq 0
\end{array}\right. \\
D \cdot \nu=\psi\left(u_{\nu}\right) \phi_{L}\left(\varphi-\varphi_{0}\right) .
\end{array}\right\} \quad \begin{aligned}
& \text { on } \Gamma_{3} . \\
&
\end{aligned}
$$

Here $\mu$ is the coefficient of friction and $R$ is a regularization operator. Finally, $\phi_{L}$ is the truncation function, used to control the roundedness of $\left(\varphi-\varphi_{0}\right)$, where $\varphi_{0}$ represents the electric potential of the foundation (see [4]). The formulation of the problem is as follows

Problem $P$. Find a displacement field $u: \Omega \rightarrow \mathbb{R}^{d}$ and an electric potential $\varphi: \Omega \rightarrow \mathbb{R}$ such that (1.1)-(1.4) hold.

Next, we derive a weak formulation of the problem $P$. To this end, we use the following functional spaces

$$
H=L^{2}(\Omega)^{d}, \quad H_{1}=H^{1}(\Omega)^{d}, \quad \mathcal{H}=\left\{\tau=\left(\tau_{i j}\right) ; \tau_{i j}=\tau_{j i} \in L^{2}(\Omega)\right\} .
$$

We also introduce the spaces for the displacement and the electric potential:

$$
V=\left\{v \in H_{1} ; v=0 \text { on } \Gamma_{1}\right\}, \quad W=\left\{\xi \in H^{1}(\Omega) / \xi=0 \text { on } \Gamma_{a}\right\} .
$$


On $V$ we consider the inner product given by $(u, v)_{V}=(\varepsilon(u), \varepsilon(v))_{\mathcal{H}}$, and let $\|\cdot\|_{V}$ be the associated norm. It follows from Korns inequality that $\|\cdot\|_{V}$ and $\|\cdot\|_{H_{1}}$ are equivalent norms on $V$. Therefore $\left(V,\|\cdot\|_{V}\right)$ is a real Hilbert space. On $W$ we consider the inner product given by $(\varphi, \xi)_{W}=(\varphi, \xi)_{H^{1}}$ for $\varphi, \xi \in W$. Then $\left(W,\|\cdot\|_{W}\right)$ is also Hilbert space. Moreover, let $K$ be the set of admissible displacements given by

$$
K=\left\{v \in V ; v_{\nu} \leq 0 \quad \text { on } \Gamma_{3}\right\} .
$$

The following assumptions are made on the given data:

$\left(\mathbf{h}_{\mathbf{1}}\right)$ - The elasticity operator $\mathfrak{F}$ is strongly monotone and Lipschitz continuous such that $\forall \xi \in$ $\mathbb{S}^{d}, x \rightarrow \mathfrak{F}(x, \xi)$ is Lebesgue measurable on $\Omega$, and $x \rightarrow \mathfrak{F}(x, 0)$ belongs to $\mathcal{H}$;

$\left(\mathbf{h}_{2}\right)$ - The piezoelectric tensor $\mathcal{E}=\left(e_{i j k}\right)$ is satisfies $e_{i j k}=e_{i k j} \in L^{\infty}(\Omega)$;

$\left(\mathbf{h}_{3}\right)$ - The electric permittivity tensor $\beta=\left(\beta_{i j}\right) \in L^{\infty}(\Omega)$ is assumed to be symmetric and $W$-elliptic;

$\left(\mathbf{h}_{4}\right)$ - The surface electrical conductivity function $\psi: \Gamma_{3} \times \mathbb{R} \rightarrow \mathbb{R}_{+}$is a Lipschitz, bounded and measurable function on $\Gamma_{3}$ for all $u \in \mathbb{R}$, which satisfies $\psi(x, u)=0$ for all $u \leq 0$;

$\left(\mathbf{h}_{\mathbf{5}}\right)$ - The coefficient of friction $\mu: \Gamma_{3} \times \mathbb{R}_{+} \rightarrow \mathbb{R}_{+}$is a Lipschitz and measurable function on $\Gamma_{3}$ for all $u \in \mathbb{R}$, which satisfies $\exists \mu^{*}>0$ such that $\mu(x, u) \leq \mu^{*}, \forall u \in \mathbb{R}_{+}$, a.e. $x \in \Gamma_{3}$;

$\left(\mathbf{h}_{\mathbf{6}}\right)$ - The density of volume forces, tractions, volume electric charges, surface electric charges and given potential have the regularity $f_{0} \in L^{2}(\Omega)^{d}, f_{2} \in L^{2}\left(\Gamma_{3}\right)^{d}, q_{0} \in L^{2}(\Omega), q_{2} \in$ $L^{2}\left(\Gamma_{b}\right)$ and $\varphi_{0} \in L^{2}\left(\Gamma_{3}\right)$;

$\left(\mathbf{h}_{7}\right)$ - The mapping $R: H_{\Gamma_{3}}^{\prime} \rightarrow L^{\infty}\left(\Gamma_{3}\right)$ is a linear and continuous function (see [5]).

Next, we define $f \in V$ and $q \in W$ as for all $v \in V$ and $\xi \in W$

$$
(f, v)_{V}=\int_{\Omega} f_{0} \cdot v d x+\int_{\Gamma_{2}} f_{2} \cdot v d a, \text { and }(q, \xi)_{W}=\int_{\Omega} q_{0} \xi d x-\int_{\Gamma_{b}} q_{2} \xi d a .
$$

Finally, we define the mappings $\ell: V \times W \times W \rightarrow \mathbb{R}$ and $j: V \times V \rightarrow \mathbb{R}$, respectively, by

$$
\begin{gathered}
\ell(u, \varphi, \xi)=\int_{\Gamma_{3}} \psi\left(u_{\nu}\right) \phi_{L}\left(\varphi-\varphi_{0}\right) \xi d a, \forall u \in V, \forall \varphi, \xi \in W, \\
j(u, v)=\int_{\Gamma_{3}} \mu\left(\left\|u_{\tau}\right\|\right)\left|R \sigma_{\nu}(u)\right|\left\|v_{\tau}\right\| d a, \quad \forall u, v \in V .
\end{gathered}
$$

Using Greens formula and the previous boundary conditions, the variational formulation for the mechanical problem $P$ is as follows: 
Problem $P V$. Find a displacement field $u \in K$ and an electric potential $\varphi \in W$ such that: $\forall v \in K$ and $\forall \xi \in W$ we have

$$
\begin{gathered}
(\mathfrak{F} \varepsilon(u), \varepsilon(v)-\varepsilon(u))_{\mathcal{H}}+\left(\mathcal{E}^{*} \nabla \varphi, \varepsilon(v)-\varepsilon(u)\right)_{L^{2}(\Omega)^{d}}+j(u, v)-j(u, u) \geq(f, v-u)_{V} \\
(\beta \nabla \varphi, \nabla \xi)_{L^{2}(\Omega)^{d}}-(\mathcal{E} \varepsilon(u), \nabla \xi)_{L^{2}(\Omega)^{d}}+\ell(u, \varphi, \xi)=(q, \xi)_{W} .
\end{gathered}
$$

\section{Existence and uniqueness result}

The main existence and uniqueness result, which we establish in this section, is the following.

Theorem 1. Under the assumption $\left(\mathbf{h}_{\mathbf{1}}\right)-\left(\mathbf{h}_{\mathbf{7}}\right)$, the problem $P V$ has at least one solution. Moreover, there exists $L^{*}$, which depends only on $\Omega, \Gamma_{1}, \Gamma_{2}, \Gamma_{3}, \mathfrak{F}, \mathcal{E}, \beta$, such that if $L_{\mu}+\mu^{*}+L_{\psi} L+$ $M_{\psi}<L^{*}$ the solution is unique.

Proof. The proof of Theorem 1 will be carried out in several steps. Let consider the product spaces $X=V \times W$ and $Y=L^{2}\left(\Gamma_{3}\right) \times L^{2}\left(\Gamma_{3}\right)$ together with the inner products

$$
\begin{gathered}
(x, y)_{X}=(u, v)_{V}+(\varphi, \xi)_{W}, \forall x=(u, \varphi), y=(v, \xi) \in X, \\
(\eta, \theta)_{Y}=(g, \lambda)_{L^{2}\left(\Gamma_{3}\right)}+(z, \zeta)_{L^{2}\left(\Gamma_{3}\right)}, \quad \forall \eta=(g, z), \theta=(\lambda, \zeta) \in Y,
\end{gathered}
$$

and the associated norms $\|\cdot\|_{X}$ and $\|\cdot\|_{Y}$, respectively. Let $U=K \times W$ be non-empty closed convex subset of $X$. We define the operator $A: X \rightarrow X$, the functions $\widetilde{j}, \widetilde{\ell}$ on $X \times X$ and the element $f_{3} \in X$ by:

$$
\begin{gathered}
(A x, y)_{X}=(\mathfrak{F} \varepsilon(u), \varepsilon(v))_{\mathcal{H}}+(\beta \nabla \varphi, \nabla \xi)_{L^{2}(\Omega)^{d}}+\left(\mathcal{E}^{*} \nabla \varphi, \varepsilon(v)\right)_{L^{2}(\Omega)^{d}}-(\mathcal{E} \varepsilon(u), \nabla \xi)_{L^{2}(\Omega)^{d}}, \\
\widetilde{j}(x, y)=j(u, v), \quad \widetilde{\ell}(x, y)=\int_{\Gamma_{3}} \psi\left(u_{\nu}\right) \phi_{L}\left(\varphi-\varphi_{0}\right) \xi d a \quad \text { and } \quad f_{3}=(f, q) \in X,
\end{gathered}
$$

for all $x=(u, \varphi)$ and $y=(v, \xi)$ in $X$. We have the following equivalence result

Lemma 2. The couple $x=(u, \varphi)$ is a solution to problem PV if and only if:

$$
(A x, y-x)_{X}+\widetilde{j}(x, y)-\widetilde{j}(x, x)+\widetilde{\ell}(x, y-x) \geq\left(f_{3}, y-x\right)_{X}, \forall y=(v, \xi) \in K \times W .
$$

The proof of this Lemma can be found in [3]. Now to prove the existence and uniqueness result of (2.5), we define two closed convex set $\mathcal{K}_{1}=\left\{g \in L^{2}\left(\Gamma_{3}\right) / g \geq 0\right.$ and $\left.\|g\|_{L^{2}\left(\Gamma_{3}\right)} \leq k_{1}\right\}$ and $\mathcal{K}_{2}=$ $\left\{z \in L^{2}\left(\Gamma_{3}\right) /\|z\|_{L^{2}\left(\Gamma_{3}\right)} \leq k_{2}\right\}$ with $k_{1}$ and $k_{2}$ to be specified. Let $\eta=(g, z) \in L^{2}\left(\Gamma_{3}\right) \times L^{2}\left(\Gamma_{3}\right)$ be given and consider the elements $f_{\eta} \in X$ given by $\left(f_{\eta}, y\right)_{X}=(f, v)_{v}+(q, \xi)_{W}-\int_{\Gamma_{3}} z \xi d a, \forall y=$ 
$(v, \xi) \in U$, define the functional $\widetilde{j}_{g}(x)=\int_{\Gamma_{3}} g\left\|v_{\tau}\right\| d a, \quad \forall x=(u, \varphi) \in U$ and consider the following intermediate Problem.

$$
\left(A x_{\eta}, y-x_{\eta}\right)_{X}+\widetilde{j_{g}}(y)-\widetilde{j}_{g}\left(x_{\eta}\right) \geq\left(f_{\eta}, y-x_{\eta}\right)_{X}, \quad \forall y=(v, \xi) \in U .
$$

Keeping in mind $\left(\mathbf{h}_{\mathbf{1}}\right)-\left(\mathbf{h}_{\mathbf{3}}\right)$ we see that $A$ is a strongly monotone and Lipschitz continuous operator. Moreover, it is easy to see that $\widetilde{j_{g}}$ is a proper, convex and continuous function on $U$. The existence and uniqueness result for (2.6) follow from standard arguments of elliptic variational inequalities (see [1]). That is, for any $\eta \in \mathcal{K}_{1} \times \mathcal{K}_{2}$. The problem (2.6) has a unique solution $x_{\eta}=\left(u_{\eta}, \varphi_{\eta}\right) \in K \times W$.

We now consider the operator $\Lambda: L^{2}\left(\Gamma_{3}\right) \times L^{2}\left(\Gamma_{3}\right) \rightarrow L^{2}\left(\Gamma_{3}\right) \times L^{2}\left(\Gamma_{3}\right)$ such that for all $\eta \in L^{2}\left(\Gamma_{3}\right) \times L^{2}\left(\Gamma_{3}\right)$, we have

$$
\Lambda \eta=\left(\mu\left(\left\|u_{\eta \tau}\right\|\right)\left|R \sigma_{\nu}\left(u_{\eta}\right)\right|, \psi\left(u_{\eta \nu}\right) \phi_{L}\left(\varphi_{\eta}-\varphi_{0}\right)\right), \quad \forall \eta \in L^{2}\left(\Gamma_{3}\right) \times L^{2}\left(\Gamma_{3}\right) .
$$

It follows from assumptions $\left(\mathbf{h}_{\mathbf{4}}\right)-\left(\mathbf{h}_{\mathbf{5}}\right),\left(\mathbf{h}_{\mathbf{7}}\right)$ that the operator $\Lambda$ is well-defined. We prove the following result

Proposition 3. If $k_{1}=c_{2} \mu^{*} c_{0} c_{*}\left(\|f\|_{V}+\|q\|_{W}+M_{\psi} L m e s\left(\Gamma_{3}\right)^{\frac{1}{2}}\right)$ and $k_{2}=M_{\psi} L \operatorname{mes}\left(\Gamma_{3}\right)^{\frac{1}{2}}$, then the operator $\Lambda$ has at least one fixed point.

Proof. We prove that

1. The mapping $\eta \rightarrow x_{\eta}$, where $x_{\eta}$ is the solution to (2.6), is weakly continuous from $L^{2}\left(\Gamma_{3}\right) \times$ $L^{2}\left(\Gamma_{3}\right)$ to $X$ and the operator $\Lambda$ is weakly continuous of $\mathcal{K}_{1} \times \mathcal{K}_{2}$ into itself.

2. $\mathcal{K}_{1} \times \mathcal{K}_{2}$ is a nonempty, convex and closed subset of $L^{2}\left(\Gamma_{3}\right) \times L^{2}\left(\Gamma_{3}\right)$. Since $L^{2}\left(\Gamma_{3}\right) \times L^{2}\left(\Gamma_{3}\right)$ is a reflexive space, $\mathcal{K}_{1} \times \mathcal{K}_{2}$ is weakly compact.

By Schauder's fixed point theorem the operator $\Lambda$ has at least one fixed point. Now, the existence part follows from the existence of the fixed point of the operator $\Lambda$.

For the uniqueness part. Let $x_{1}=\left(u_{1}, \varphi_{1}\right), x_{2}=\left(u_{2}, \varphi_{2}\right) \in X$ be two solutions of problem (2.5) we establish after some algebra the following estimate

$$
\left\|x_{1}-x_{2}\right\|_{X}^{2} \leq c\left(L_{\mu}+\mu^{*}+L_{\psi} L+M_{\psi}\right)\left\|x_{1}-x_{2}\right\|_{X}^{2} .
$$

Let $L^{*}=1 / c$, then if $L_{\mu}+\mu^{*}+L_{\psi} L+M_{\psi}<L^{*}$ therefore $x_{1}=x_{2}$.

\section{Error estimates for the numerical approximation}

In this section, we study the finite element approximation of the variational problem $P V$. Assume $\Omega$ is a polygonal domain, let $\tau^{h}$ be a regular family of triangular finite element partitions of $\bar{\Omega}$ that are compatible with the partition of the boundary decompositions $\Gamma=\Gamma_{1} \cup \Gamma_{2} \cup \Gamma_{3}$ and $\Gamma=\Gamma_{a} \cup \Gamma_{b} \cup \Gamma_{3}$, that is, any point when the boundary condition type changes is a vertex of the 
partitions, then the side lies entirely in $\bar{\Gamma}_{1} \cup \bar{\Gamma}_{2} \cup \bar{\Gamma}_{3}$, and $\bar{\Gamma}_{a} \cup \bar{\Gamma}_{b} \cup \bar{\Gamma}_{3}$. Corresponding to each partition $\tau^{h}$. We denote by $\mathbb{P}_{1}\left(\Omega^{e}\right)$ the space of polynomials of global degree less or equal to one in $\Omega^{e}$. Let us consider two finite-dimensional spaces $V^{h} \subset V$ and $W^{h} \subset W$, approximating the spaces $V$ and $W$, respectively, that is

$$
\begin{aligned}
& V^{h}=\left\{v^{h} \in C(\bar{\Omega})^{d}, v_{/ \Omega^{e}}^{h} \in \mathbb{P}_{1}\left(\Omega^{e}\right)^{d}, \Omega^{e} \in \tau^{h}, v^{h}=0 \text { on } \bar{\Gamma}_{1}\right\}, \\
& W^{h}=\left\{\psi^{h} \in C(\bar{\Omega}), \psi_{/ \Omega^{e}}^{h} \in \mathbb{P}_{1}\left(\Omega^{e}\right), \Omega^{e} \in \tau^{h}, \psi^{h}=0 \text { on } \bar{\Gamma}_{a}\right\} .
\end{aligned}
$$

Here $h>0$ is a discretization parameter. Moreover let us consider the nonempty finite-dimensional closed convex sets of admissible displacements with $V^{h}$, defined by $K^{h}=K \cap V^{h}$. i.e. $K^{h}=$ $\left\{v^{h} \in V^{h}, v_{\nu}^{h} \leq 0\right.$ on $\left.\bar{\Gamma}_{3}\right\}$. We consider the following discrete approximation of problem $P V$ :

Problem $P V^{h}$. Find a discrete displacement field $u^{h} \in K^{h}$ and a discrete electric potential $\varphi^{h} \in W^{h}$ such that

$$
\begin{aligned}
& \left(\mathfrak{F} \varepsilon\left(u^{h}\right), \varepsilon\left(v^{h}\right)-\varepsilon\left(u^{h}\right)\right)_{\mathcal{H}}+\left(\mathcal{E}^{*} \nabla \varphi^{h}, \varepsilon\left(v^{h}\right)-\varepsilon\left(u^{h}\right)\right)_{L^{2}(\Omega)^{d}}+j\left(u^{h}, v^{h}\right)-j\left(u^{h}, u^{h}\right) \\
& \geq\left(f, v^{h}-u^{h}\right)_{V}, \quad \forall v^{h} \in K^{h} . \\
& \left(\beta \nabla \varphi^{h}, \nabla \xi^{h}\right)_{L^{2}(\Omega)^{d}}-\left(\mathcal{E} \varepsilon\left(u^{h}\right), \nabla \xi^{h}\right)_{L^{2}(\Omega)^{d}}+\ell\left(u^{h}, \varphi^{h}, \xi^{h}\right)=\left(q, \xi^{h}\right)_{W}, \forall \xi^{h} \in W^{h} .
\end{aligned}
$$

Using the assumptions of Theorem 1, it can be shown that Problem $P V^{h}$ has a unique solution $\left(u^{h}, \varphi^{h}\right) \in K^{h} \times W^{h}$. Our interest lies in estimating the numerical errors. We first derive a Céa's type inequality.

Theorem 4. Let us denote by $(u, \varphi)$ and $\left(u^{h}, \varphi^{h}\right)$ the respective solutions to problem $P V$ and $P V^{h}$. Under the assumptions of Theorem 1 with the same value of $L^{*}$, the following error estimates are obtained for all $v^{h} \in K^{h}$ and $\psi^{h} \in W^{h}$,

$$
\begin{aligned}
& \left\|u-u^{h}\right\|_{V}+\left\|\varphi-\varphi^{h}\right\|_{W} \leq c \inf _{\left(v^{h}, \xi^{h}\right) \in K^{h} \times W^{h}}\left\{\left\|u-v^{h}\right\|_{V}+\left\|\varphi-\xi^{h}\right\|_{W}\right. \\
& +\left\|u-v^{h}\right\|_{L^{2}\left(\Gamma_{3}\right)^{d}}+\left\|\varphi-\xi^{h}\right\|_{L^{2}\left(\Gamma_{3}\right)}+\left(\|\mathfrak{F} \varepsilon(u)\|_{\mathcal{H}}^{\frac{1}{2}}+\left\|\mathcal{E}^{*} \nabla \varphi^{h}\right\|_{\mathcal{H}}^{\frac{1}{2}}+\|f\|_{V}^{\frac{1}{2}}\right)\left\|u-v^{h}\right\|_{V}^{\frac{1}{2}} \\
& \left.+\left(\left\|R \sigma_{\nu}(u)\right\|_{L^{\infty}\left(\Gamma_{3}\right)}\left\|\mu\left(\left\|u_{\tau}\right\|\right)\right\|_{L^{2}\left(\Gamma_{3}\right)}\right)^{\frac{1}{2}}\left\|u-v^{h}\right\|_{L^{2}\left(\Gamma_{3}\right)^{d}}^{\frac{1}{2}}\right\},
\end{aligned}
$$

where $c>0$ is independent of $h$.

The proof of Theorem 4 is done by using a properties $\left(\mathbf{h}_{\mathbf{1}}\right)-\left(\mathbf{h}_{\mathbf{7}}\right)$ and after some tedious algebraic manipulations. The inequality (3.3) is a basis for deriving error estimation and convergence analysis. In an analogous way, if we also suppose that $\sigma_{\tau} \in L^{2}\left(\Gamma_{3}\right)^{d}$ and using the classical results of interpolation (cf. [2]), we have the following result

Theorem 5. Under the assumptions of Theorem 1 with the same value of $L^{*}$, assume additionally $\sigma_{\tau} \in L^{2}\left(\Gamma_{3}\right)^{d}$. Then for some constant $c>0$, we have

$$
\begin{aligned}
\left\|u-u^{h}\right\|_{V}+\left\|\varphi-\varphi^{h}\right\|_{W} \leq & c h\left\{|u|_{H^{2}(\Omega)^{d}}+|\varphi|_{H^{2}(\Omega)}+h|u|_{H^{2}\left(\Gamma_{3}\right)^{d}}+h|\varphi|_{H^{2}\left(\Gamma_{3}\right)}\right. \\
& \left.+\left(\left\|\sigma_{\tau}\right\|_{L^{2}\left(\Gamma_{3}\right)^{d}}+\left\|R \sigma_{\nu}(u)\right\|_{L^{\infty}\left(\Gamma_{3}\right)}\left\|\mu\left(\left\|u_{\tau}\right\|\right)\right\| \|_{L^{2}\left(\Gamma_{3}\right)^{2}}\right)^{\frac{1}{2}}|u|_{H^{2}\left(\Gamma_{3}\right)^{d}}^{\frac{1}{2}}\right\} .
\end{aligned}
$$




\section{References}

[1] H. Brezis. Equations et inéquations non linéaires dans les espaces vectoriels en dualité. Annales Inst. Fourier, 18 (1968), 115-175.

[2] P. G. Ciarlet. The finite element method for elliptic problems. North-Holland, Amsterdam, 1978.

[3] M. Sofonea, EL-H. Essoufi. A Piezoelectric contact problem with slip dependent coefficient of friction. Math. Model. Anal., 9 (2004), 229-242.

[4] Z. Lerguet, M. Shillor, M. Sofonea. A frictional contact problem for an electro-viscoelastic body. Electronic journal of differential equations, 2007 (2007), No. 170, 1-16.

[5] A. Touzaline. A Quasistatic unilateral contact problem with slip-dependent coefficient offriction for nonlinear elastic materials. Electronic journal of differential equations, 2006 (2006), No. 144, 1-14. 